\title{
ISOMORPHISMS BETWEEN REPRESENTATIONS OF ALGEBRAS ${ }^{1}$
}

\author{
Manuel SaOkín
}

A la memoria de Pere Menal, guía, compañero y amigo

\begin{abstract}
In this paper we study the precise relation between two representations of a given split basic finite dimensional algcbra $A$ as a factor of the free path algebra over its quiver (A). After defining the notion of strongly acyclic quiver, we apply the results obtained to devolop a method of calculating the group $A u t(A) / \operatorname{Inn}(A)$ in the case when $(A)$ is strongly acyclic.
\end{abstract}

The representation of algebras by quivers and relations has a long tradition. It is well-known that if $A$ is a finite dimensional algebra which is split over the field $K$ (i.e., $A=B \oplus J(A)$ as a $K$-vector space, wherc $B$ is a subalgebra of $A$ isomorphic to a direct product of full matrix algebras over $K$ and $J(A)$ is the Jacobson radical of $A$ ), then $A$ is Morita equivalent to an algebra, usually called its basic algebra, that is isomorphic to a factor of the path algebra $K[\Gamma]$ of its quiver $\Gamma$ (sec, e.g., Section 27 of ' 1 ! and [2]). The study of the connection between any two factors of $K[\Gamma]$ that are both isomorphic to the basic algebra of $A$ is, together with some consequences of it, the aim of this work. This study is divided into two parts. In the present article we study the connection itself (Theorem 3 and Corollary 5 ) and use what is found to approach the group of automorphisms of $A$, when the quiver is of a special type (Theorem 7). We shall show further consequences of this connection in a subsequent work, that we hope will be soon in preprint.

In what follows, unless otherwise stated, $A$ will be a split finite dimensional algebra which is basic, i.e., the above mentioned subalgebra $B$ is a direct product of copies of $K$. Since we want to emphasizc a

\footnotetext{
${ }^{1}$ Most of this work was prepared while the anthor was visiting the University of lowa, in Iowa City, U.S.A. Supported by the DGICYT.
} 
certain uniqueness of $B$ in that decomposition of $A$, we will assume a condition on $K$, like that of being a pcrfect field, that guarantees that the Wedderburn-Malcev's Theorem (see 11.6 of [3]) applies. For all the terminology concerning the relation between a finite dimensional algebra and its associated basic one or, more generally, between an Artinian ring and its associated basic one, we refer the reader to ([1, Section 27]). When dealing with maps between algebras, homomorphism and automorphism will always mean $K$-homomorphism and $K$-autonorphism (i.e., inducing the identity on $K$ ), respectively. In reference to the representation of $A$ by its quiver and relations, we follow the terminology of Gabriel ([2]), except for the composition of arrows. We will write $\alpha \beta$ for a paih.$\stackrel{\alpha}{\rightarrow} \cdot \stackrel{\beta}{\mapsto}$. We will denote by $\Gamma(A)$, or simply $\Gamma$ if no confusion appears, the quiver of $A$ and by $V(\Gamma)$ (resp. $A(\Gamma)$ ) the set of vertices (resp. arrows) of $\Gamma$. The symbol $K[\Gamma]$ will stand for the corresponding free path algcbra and $J$ for the ideal of $K[\Gamma]$ consisting of all linear combinations of paths of length $\geq 1$. It is well-known (see [2]) that $A$ is isomorphic to a quotient of $K[\Gamma]$ by an ideal $I$ satisfying $J^{m} \subseteq I \subseteq J$, for some $m \geq 1$. Every such an ideal, which is usually not unique, is called an adequate ideal for $A$ in $K[\Gamma]$. Being $K[\Gamma] / J^{m}$ a finite dimensional algebra, hence Artinian, every adequate ideal $I$ is finitely generated modulo $J^{m}$ and from that follows easily that $I$ is itself finitely generated. Every finite set $\rho$ of generators for an adequate ideal is called an adequate set of relations for $A$ in $K[\Gamma]$. For our purposes, it will not be restrictive to assume that $A$ is also indecomposable (as a proper direct product of algebras) or, equivalently, that $\Gamma$ is connected (i.e. there is a not necessarily oriented path between any two vertices). In that case, either $A$ is isomorphic to $K$ or, otherwise, every adequatc icleal for $A$ in $K\left[\Gamma^{2}\right]$ is contained in $J^{2}$. We will always assume this situation in the sequel. On the other hand, we shall denote by $\mathcal{P}_{n}$, for every $n \geq 0$, the set of all paths of length exactly $n$ in $\Gamma$. By convention, a path of length 0 is just a vertex.

In the following result and later on in these notes, we will sometimes use the notation $x^{\sigma}$ to denote the action of certain autornorphism on the element $x$ and $X^{\sigma}$ to denote the image of the subset $X$ by $\sigma$.

Lemma 1. Let $I$ be an adequale ideal for $A$ in $K[G]$. For every basic set of idempotents $\left\{e_{1}, \ldots, e_{7 t}\right\}$ of $A$, there is a surjective homomorphism of algebras $p: K[\Gamma] \mapsto A$ such that $\operatorname{ker} p=I$ and, as sets, $p(V(\Gamma))=$ $\left\{e_{1}, \ldots, e_{n}\right\}$.

Proof: Since $I$ is an adequate ideal for $A$, we have an isomorphism of algebras $h: K[\Gamma] / I \mapsto A$ and $h(\overline{V(\Gamma)})$ is a basic set of idempotents for $A$, where $\overline{V(\Gamma)}$ denotes the set of classes of the vertices modulo 1 . If we 
denote by $B$ the subalgebra of $A$ generated by $h(\overline{V(\Gamma)})$ and by $B^{*}$ that generated by $\left\{e_{1}, \ldots, e_{n}\right\}$, then we know that $A=B \oplus J(A)=B^{*} \Theta J(A)$. By the Wedderburn-Malcev Theorem (see, e.g., [3, Theor. 11.6]), there is a inner automorphism $\sigma$ of $A$ such that $B^{\sigma}=B^{*}$. But then $h(\overline{V(\Gamma)})^{\sigma}$ is a basic sei of idempotents of $B^{*}$ that must necessarily coincide with $\left\{e_{1}, \ldots, e_{n_{2}}\right\}$. Therefore the composition $K[\Gamma] \stackrel{\pi}{\rightarrow} K[\mathrm{\Gamma}] / I \stackrel{h}{\leftrightarrow} A \stackrel{o}{\mapsto} A$, where $\pi$ is the carnonical projection, is the desired surjective homomorphism of algebras.

Remark 2. According to the above lemma, we do not need to worry about the basic set of idempotents of $A$ since, up to a permutation in the set, all the representations of $A$ in the form $K[\mathrm{r}] / J$ are obtained by sending the vertices of $\Gamma$ to that set.

Definition. An automorphism of the quiver $\Gamma$ is a pair of bijective maps, derioted by the same letter $\sigma, V(\Gamma) \stackrel{\sigma}{\sim} V(\Gamma)$ and $A(\Gamma) \stackrel{\sigma}{\sim} A(\Gamma)$, so that if $\alpha$ is an arrow from $\nu$ to $\omega(\nu, \omega \in V(\Gamma))$ then $\alpha^{\sigma}$ is an arrow from $\nu^{\sigma}$ to $\omega^{\sigma}$.

It is obvions that an automorphism of the quiver induces an automorphism of the path algebra $K[\Gamma]$. 'To avoid too much notation, we denote it also by $\sigma$. If now $I$ is an adequate ideal for $A$ in $K[\Gamma]$, then $I^{\sigma}$ is another adequate ideal and $\sigma$ induces an isomorphism of adequate representations $K[\Gamma] / I \cong K[\Gamma] / I^{\sigma}$. Any isomorphism of adequate representations obtained in this manner will be referred as induced from the quiver.

In order to describe completely the isomorphisms between adequate representations of $A$, we need the following definition, in which $A(\nu, \omega)$ denotes the set of arrows in $\Gamma$ starling at the vertex $\nu$ and ending at $\omega$.

Definition. A change of variables in $K[\Gamma]$ is an algebra homomorphism $f: K[\Gamma] \mapsto K\left[\left[^{\top}\right]\right.$ such that:

i) $\int$ induces the identity in $V(\Gamma)$.

ii) If $A(\nu, \omega)=\left\{\alpha_{1}, \ldots, \alpha_{m_{\nu \omega}}\right\}$ (an ordering of the $\alpha_{k}$ 's being fixed), then $f\left(\alpha_{l}\right)=\sum_{k=1}^{m_{k_{w}}} \lambda_{k l} \alpha_{k}$ (modulo $J^{2}$ ) for each $l$, where, for each pair of vertices $\nu, \omega$, the $m_{\nu_{w}} \times m_{\nu w}$ matric (with coeflicients in K) $\Lambda=\left(\lambda_{k t}\right)$ is invertible.

Note that, if condition i) above holds, then condition ii) is equivalent to the following:

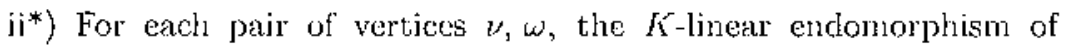
$\nu J \omega / \nu J^{2} \omega$ induced by $f$ is an automorphism.

Now comes the main result of this first part, where, for every ideal $I$ of $K[\Gamma], V(\Gamma)+I / I$ staurls for the projection of $V(\Gamma)$ via the canonical 
projection $p: K[\Gamma] \leftrightarrow K[\Gamma] / I$.

Theorem 3. Let $A$ be of Loewy length $m, I$ an adequate ideal for $A$ in $K[\Gamma]$ and $L$ an arbilrary ideal of $K[\Gamma]$. The following assertions are equivalent for a homomorphism of algebras $\phi: K[\Gamma] / I \mapsto K[\Gamma] / L$ :

a) $\phi$ is an isomorphism of $K$-algebras such that $\phi(V(\Gamma)+I / I)=$ $V(\Gamma)+L / L$

b) $J^{k} \subseteq L$, for some integer $k \geq m$, and there are an automorphism $\sigma$ of $k[\Gamma]$ induced from the quiver and a change of variables $f$ in $K|\Gamma|$ such that $I=\sigma^{-1}\left(f^{-1}(L)\right)$ and $\phi$ is induced by $f \circ \sigma$.

b) As in b), but with $I=f^{-1}\left(\sigma^{-1}(L)\right)$ and $\sigma \circ f$ instead of $I=$ $\sigma^{-1}\left(f^{-1}(L)\right)$ and $f \circ \sigma$, respectively.

c) There are $\sigma$ and $f$ as in b) such that $L=f\left(I^{\sigma}\right)+J^{k}$, for some $k \geq m$, and $\phi$ is induced by $f \circ \sigma$.

c) As in c), but with $L=f(I)^{\sigma}+J^{k}$ and $\sigma \circ f$ instead of $L=$ $f\left(I^{\sigma}\right)+J^{k}$ and $f \circ \sigma$, respectively.

Before tackling the proof of this theorem, we give a result upon which we shall lean.

Lemma 4. If $f$ is a change of variables in $K[\Gamma]$, then the induced homomorphism of algebras $\hat{f}: K[\Gamma] / J^{r} \mapsto K[\Gamma] / J^{r}$ is an antomorphism, for every $r \geq 1$.

Proof: It will be enough to prove that, modulo $J^{r}$, every path is in the image of $f$. The result is obvious, by the definition of a change of variables, for $r=1,2$. If $r \geq 3$ we proceed by decreasing induction on the length $s$ of the path $p=\alpha_{1} \ldots \alpha_{s}$ in question. If $s \geq r$ it is evident, since $p \equiv 0\left(\bmod J^{\tau}\right)$. Suppose now that $s \leq r-1$ and that every path of length $\geq s+1$ is in the inage of $f$ modulo $J^{r}$. By the definition of a change of variables again, every arrow $\alpha_{i}$ appearing in $p$ is in the image of $f$ modulo $J^{2}$. From that follows that $p$ is in the image of $f$ modulo $y^{s+1}$, and the induction hypothesis applies to show that $p$ is in the image of $f$ modulo $J^{r}$, as desired.

Proof of Theorem 3: a) $\Rightarrow$ b). Since $\phi(V(\Gamma)+I / I)=V(\Gamma)+L / L$ we have a permutation $\sigma$ of $V(\Gamma)$ such that $\phi(\nu+I)=\nu^{\sigma}+L$, for each vertex $\nu$. It is clear that the number of arrows from $\nu$ to $\omega$ coincicles with that of arrows from $\nu^{\sigma}$ to $\omega^{\sigma}$, so that $\sigma$ can be extended to a not necessarily unique automorphism of the quiver $\Gamma$. We fix one among the possible automorphisms of $\Gamma$ that extend $\sigma$ and, as usual, we also denote by $\sigma$ the corresponding automorphism induced in $K\left[\mathrm{I}^{\prime}\right]$. Now we consider the decomposition $\phi=\phi \circ \bar{\sigma}^{-1} \circ \bar{\sigma}$, where $\bar{\sigma}: K[\Gamma] / I \mapsto K\left[\Gamma_{\mathrm{j}} / I^{\sigma}\right.$ is the 
isomorphism induced by $\sigma$. Since clearly $\phi \circ \bar{\sigma}^{-1}\left(\nu+I^{\sigma}\right)=\nu+L$, by replacing $\phi$ by $\phi \circ \bar{\sigma}^{-1}$ and $I$ by $I^{\sigma}$ if necessary, it is not restrictive to assume that $\phi(\nu+I)=\nu+L$, for every vertex $\nu$. All we have to prove now is that $J^{k} \subseteq L$, for some $k \geq m$, and $\phi$ is induced by a change of variables $f$ such that $I=f^{-1}(L)$. To prove the inclusion it is enough to check that $J^{m} \subseteq L$, but this follows in a straightforward way from the fact that $K[I] / L$ is isomorphic to $A$, by assumption, and hence the dimensions and Loewy lengths of both algebras must coincide. On the other hand, if $\alpha \in A(\nu, \omega)$ then $\alpha+I \in(\nu+I)(J / I)(\omega+I)$, so that $\phi(\alpha+I) \in(\nu+L)(J / L)(\omega+L)=\nu / J \omega+L / L$. Let us fix an element $\eta(\alpha)$ in $\nu J \omega$, i.e. a linear combination of paths of length $\geq 1$ starting at $\nu$ and ending at $\omega$, such that $\phi(\alpha+I)=\eta(\alpha)+L$ and do the same for all vertices $\nu, \omega$ and all arrows in $A(\nu, \omega)$. The freeness of the path algebra allows us to extend the assignments $\alpha \mapsto \eta(\alpha)$ to a homomorphism of algebras $f: K[\Gamma] \mapsto K[\Gamma]$ that induces $\phi: K[\Gamma] / I \mapsto K[\Gamma] / L$. It only remains to prove that $f$ is actually a change of variables. But that is easy, because the inverse $\phi^{-1}$ is also induced by a homomorphism of algebras $g: K[\Gamma] \nrightarrow K[\Gamma]$ that is defined in the same way that $f$ was defined from $\Phi$. It is a mere rontine to check that the $K$-linear endomorphisms of $\nu, J \omega / \nu J^{2} \omega$ induced by $f$ and $g$ are inverse from cach other, thus proving that $f$ (and also $g$ ) is a change of variables.

a) $\left.\Rightarrow b^{1}\right)$ As the above implication, but considering the decomposition $\phi=\bar{\sigma} \circ \bar{\sigma}^{-1} \circ \phi$ instead of the above taken.

b) $\Rightarrow$ c) From b) follows at once that $f\left(I^{\sigma}\right)+J^{k} \subseteq L$ and $\phi$ is induced by $f \circ \sigma$. On the other hand, by considering the isomorphism of algebras $f: K[\Gamma] / J^{k} \rightarrow K[\Gamma] / J^{k}$ induced by $f$, it follows from Lemma 4 that $\hat{f}\left(\hat{f}^{-1}\left(L / J^{k}\right)=L / J^{k}\right.$ and hence $f\left(f^{-1}(L)\right)+J^{k}=L$. But $f^{-1}(L)$ is $I^{\sigma}$ and thus we are done.

b') $\left.\Rightarrow e^{\prime}\right)$ Completely analogous to the above one, by making the suitable changes.

c) $\Rightarrow$ a) Fron c) we derive a homomorphism of algebras $\bar{f}: K[\Gamma] / I^{\sigma} \rightarrow$ $K[\Gamma] / L$. If now $p_{1}$ and $p_{2}$ are the canonical projections of $K[\Gamma] / J^{k}$, where $k$ is the integer given by $\mathrm{c}$, onto $K[\Gamma] / I^{\sigma}$ and $K[\Gamma] / L$, respectively, we have that $p_{2} \circ \hat{f}=\bar{f} \circ p_{1}$, from which we deduce that $\bar{f}$ is surjective. On the other hand, its kernel is $f^{-1}(L) / I^{\sigma}$ and from the fact that $f$ is monic follows that $I^{\sigma} / J^{k}=f^{-1}\left[f\left(I^{\sigma}\right)+J^{k}\right] / J^{k}==f^{-1}(L) / J^{k}$. 'Therefore $\vec{f}$ is also injective and, since $\phi=\bar{f} \circ \bar{\sigma}, \sigma$ is an isomorphism.

(') $\Rightarrow$ a) As c) $\Rightarrow$ a) with the appropriate changes. 
As a consequence of the above theorem, it will be easy to express any adequate ideal for $A$ in terms of a given adequate set of relations. Let us recall first that the quiver of $A$ is said to be acyclic in case it has neither loops nor oriented cycles.

Corollary 5. Let $A$ have Loewy length $m$ and $\rho$ be an adequate set of relations for $A$ in $K[\Gamma]$. For an ideal $L$ of $K[\Gamma]$ the following assertions are equivalent:

a) $L$ is adequate for $A$.

b) There exist an integer $k \geq m$ and $f, \sigma$ as in the theorem such that $L$ is generated (as an ideal of $K[\Gamma] !)$ by $f\left(\rho^{\sigma}\right) \cup \mathcal{P}_{k}$ (or by $\left.f(\rho)^{\sigma} \cup \mathcal{P}_{k}\right)$.

c) There exist $f$ and $\sigma$ as in the theorem such that, for every $k \geq$ $m, L$ is generated by $f\left(\rho^{\sigma}\right) \cup \mathcal{P}_{k}$ (or by $f(\rho)^{\sigma} \cup \mathcal{P}_{k}$ ).

d) There exist $f$ and $\sigma$ as in the theorem such that $L$ is generated by $f\left(\rho^{\sigma}\right) \cup \mathcal{P}_{m}$ (or by $\left.f(\rho)^{\sigma} \cup \mathcal{P}_{m}\right)$.

If, moreover, $A$ has an acyclic quiver, then the above conditions are equivalent to the simpler one:

e) There exist $f$ and $\sigma$ as in the theorem such that $J$ is generated by $f\left(\rho^{\sigma}\right)$.

Proof: Let $I=\langle\rho\rangle$ be the ideal of $K[\Gamma]$ generated by $\rho$. It is clear that, for $f$ and $\sigma$ as in the theorem, $f\left(I^{\sigma}\right)+J^{k}$ (resp. $f(I)^{\sigma}+J^{k}$ ) is just the ideal of $K[\Gamma]$ generated by $f\left(\rho^{\sigma}\right) \cup \mathcal{P}_{k}$ (resp. $f(\rho)^{\sigma} \cup \mathcal{P}_{k}$ ), for every $k \geq m$. With that in mind, the equivalence of a), b), c) and d) follows in a straightforward way from Theorem 3 and Lemma 1 , once we notice that, in the proof of a) $\Rightarrow$ b) in that theorem, we actually proved statement b) for every $k \geq m$. On the other hand, $A$ has an acyclic quiver if and only if $J^{k}=0$ for some $k \geq 1$. From it follows the equivalence with $e)$ in this particular situation.

Examples 6. a) When $A$ does not have an acyclic quiver in the above corollary, $\mathcal{P}_{m}$ is necessary to guarantee that $J$ is an adequate ideal. This can be seen with the simple example $A=K[X] /\left\langle X^{3}\right\rangle$, which has a quiver consisting of a unique vertex and a loop, which is denoted by $X$ in the sequel. Obviously $\rho=\left\{X^{3}\right\}$ and the change of variables $f$ taking $X$ to $X+X^{2}$ yiekds $f(\rho)=\left\{\left(X+X^{2}\right)^{3}\right\}$, that generates an ideal of $K[\Gamma]=K[X]$ containing no power of $J=\langle X\rangle$. However, as a confirmation of condition c) of the corollary, $f(\rho) \cup\left\{X^{k}\right\}$ generates the ideal $\left\langle X^{3}\right\rangle$, for every $k \geq 3$ (which is the Loewy length of $A$ ). 
b) Let, $A$ have quiver

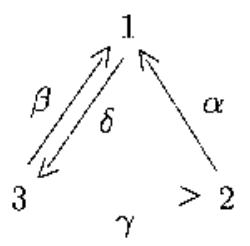

and relations $\rho=\{\beta \delta, \gamma \alpha \delta, \alpha \delta \beta-\alpha \delta \gamma \alpha\}$. It is no hard to see that its Loewy length is 5 . If now we apply the change of variables $f$ that takes $\beta \nrightarrow \beta+\gamma \alpha$ and fixes all the otler arrows, then we see that $f(\rho)=\{\beta \delta+\gamma \alpha \delta, \gamma \alpha \delta, \alpha \delta \beta\}$ so that the ideal $L$ of $K[\Gamma]$ generated by $f(\rho)$ can be generated by $\{\beta \delta, \gamma \alpha \delta, \alpha \delta \beta\}$ as well. With this and the previous corollary we can already assert that $A$ is monomial algebra, a fact not easily deducible from the first representation given for $A$. In a further step, one can easily check that every path of length 5 in $\Gamma$ is in $L$, thus proving that $A \cong K[\Gamma] / L$.

By using Lemma 1 and Theorem 3 , one can deduce, in a straightforward way, that every autonorphism $\phi$ of $A$ that leaves invariant (although not necessarily fixing its elernents) a given basic set of idempotents (i.e. $\left.\phi\left(\left\{e_{1}, \ldots, e_{n}\right\}\right)=\left\{e_{1}, \ldots, e_{n}\right\}\right)$ is induced by a composition $f \circ \sigma$ or $\sigma \circ f$, where $\sigma$ is an automorphism of $K\left[I^{\prime}\right]$ induced from the quiver and $f$ is a change of variables and, for certain adequate ideal $I$ of $K[\Gamma], f\left(I^{\sigma}\right)$ (or $\left.f(I)^{\sigma}\right)$ in included in $I$. Since, by the WedderburnMalcev Theoren, up to composition by a imner automorphism all the automorphisms of $A$ lcave invariant a given basic set of idempotents, it is then reasonable to expect, in particular cases, some information about we group $A$ ut $(A) / \operatorname{mn}(A)$ coming from our Theoren 3 . That is our next goal.

Let us introduce some notation. We will write $S_{r}$ for the group of all permutalions $\sigma$ of the set $V(\Gamma)$ satisfying that (\# arrows $\nu \longmapsto \omega$ ) $=$ (\# arrows $\nu^{\sigma} \mapsto \omega^{\sigma}$ ), for every pair of vertices $\nu, \omega$. Intuitively: $S_{l^{n}}$ is the group of all permutations of $V(\Gamma)$ which potentially can be extended to automorphisms of $\Gamma$. We will denote by $V_{1}$, the set of all changes of variables in $K[\Gamma]$ and, if $\rho$ is an adequate set of relations for $A$ in $K[\Gamma], V_{(\Gamma, \rho)}$ will stand for the subset $\left\{f \in V_{\Gamma} / f(\rho) \subseteq\langle\rho\rangle\right\}$ (one should notice that $V_{(r, p)}$ actually depends on $\langle\rho\rangle$ rather then $\rho$ itself). $V_{\Gamma}$ is a semigroup with the composition of maps as operation and $V_{(\Gamma, \rho)}$ is a subsemigroup. Moreover, we have a canonical semigroup homomorphisn $V_{(\Gamma, p)} \mapsto \operatorname{Aut}(A\rangle=\operatorname{Aut}(K[\Gamma] /\langle\rho\rangle)$ whose image turns out to be a subgroup of the $\operatorname{Aut}(A)$, by Theoren 3. We will denote by $i_{p}$ to the composition of the latter homomorphism followed by the 
Canonical projection $\pi: \operatorname{Aut}(A) \nrightarrow \operatorname{Aut}(A) / \operatorname{Inn}(A)$. We shall introduce now a certain type of algebras for which the technics developed here will help determine $\operatorname{Aut}(A) / \operatorname{Inn}(A)$.

Definition. The quiver $\Gamma$ of $A$ will be said strongly acyclic if it satisfies the following property:

(\&) For all $\nu, w \in V(\Gamma)$, if there is an arrow $\nu \mapsto \omega$, then there is no path of length $\geq 2$ from $\nu$ to $\omega$.

Theorem 7. Let A have a strongly acyclic quiver. The following properties hold:

a) $V_{\Gamma}$ is a group, which is isomorphic to $\prod_{\nu, \omega \in V(\Gamma)} G L_{7 n_{u \omega}}(K)$, where $m_{\nu \omega}$ is the number of arrows $\nu \longmapsto \omega$, for all $\nu, \omega \in V(\Gamma)$.

b) $V_{(\Gamma, \rho)}$ is a subgroup of $V_{\Gamma}$.

c) $i_{\rho}$ is an injective map.

d) There is an exact sequence of groups and group homomorphisms

$$
1 \mapsto V_{(\boldsymbol{r}, \rho)} \mapsto \operatorname{Aut}(A) / \operatorname{Inn}(A) \mapsto S_{\mathrm{r}}
$$

Proof: If $\alpha \in A(\nu, \omega)$ and $f$ is a change of variables in $K[\Gamma]$ : the fact that $\Gamma$ is strongly acyclic implies that $f(\alpha)$ must be necessarily a linear combination of arrows $\nu \mapsto \omega$. If we consider the vector subspace $V_{\nu w}$ of $K[\Gamma]$ generated by $A(\nu, \omega)$, it is clear that $f$ is completely determined by its components $f_{\nu \omega}: V_{\nu \omega \omega} \mapsto V_{\nu \omega}$, i.e, the restrictions of $f$ to these subspaces, so that the assignment $f \rightarrow\left(f_{\nu \omega}\right) \in \prod G L\left(V_{\nu \omega}\right)$ is a bijective map. It is routine to check that it preserves the group operations and thereby a) holds.

To prove b) we should observe that if $f \in V_{\langle(r, \rho)}$ then $f$ induces an automorphism $\phi$ in the algebra $K[\Gamma] /\langle\rho\rangle$ whose inverse $\phi^{-1}$ is also induced by a $g \in V_{\left(\Gamma_{1}, \rho\right)}$ due to the proof of Theorem 3. The strong acyclic nature of $\Gamma$ implies that $g=f^{-1}$.

Let us consider now a $f \in V_{(\Gamma, p)}$ such that the induced automorphism $\bar{f}: K[\Gamma] /\langle\rho\rangle \mapsto K[\Gamma] /\langle\rho\rangle$ is inner and let $u$ be an element of $A=$ $K\left[I^{2}\right] /\langle\rho\rangle$ such that $\bar{f}(a)=u a u^{-1}$, for every $a \in A$. If $\alpha \in A(\nu, \omega)$ and $A(\nu, \omega)=\left\{\alpha_{1}=\alpha, \alpha_{2}, \ldots, \alpha_{m_{\nu \omega}}\right\}$, then $f(\alpha)=\sum_{k=1}^{m_{\nu \omega}} \lambda_{k} \alpha_{k}$, with $\lambda_{k} \in K$ for each $k$. By writing a bar to denote the class modulo $\langle\rho\rangle$, we have $u \bar{\alpha} u^{-1}=\sum \lambda_{k} \bar{\alpha}_{k}$ and so $(0 \neq) u \bar{\alpha}=\sum \lambda_{k} \bar{\alpha}_{k} u$. If we view $u$ as the class modulo $\langle\rho\rangle$ of a linear combination of paths in $\Gamma$ and a coefficient, say, $\lambda_{k}$ is nonzero, then a careful look at the latter equality tells us that 
there is a path of length $\geq 1$ in $\Gamma$ that starts with the arrow $\alpha_{k}$ and ends with the arrow $\alpha$. That $\mathrm{F}$ is acyclic (it does not have to be strongly acyclic here) implies that it can only occur when $\alpha_{k}=\alpha$ and the path has length exactly 1 . From this follows at once that $\lambda_{k}=0$ when $k \neq 1$ and $\lambda_{1}=1$, thus implying that $f$ is the identity map and proving $c$ ).

Finally, for d), we should recall that every clement of $\operatorname{Aut}(A) / \operatorname{mn}(A)$ is represented by a $\phi \in A u t,(A)$ that leaves invariant $\left\{e_{\nu}=\nu+\langle\rho\rangle \mid \nu \in\right.$ $V(\Gamma)\}$. If we assign to that $\phi$ the permutation $\sigma$ of $V(\Gamma)$ defined by $\phi\left(e_{\nu}\right)=e_{\sigma(\omega)}$, then (\# arrows $\left.\nu \mapsto \omega\right)=$ (\# arrows $\nu^{\sigma} \mapsto \omega^{\sigma}$ ), for each pair for vertices $\nu, \omega$. We claim that this assignment defines a group homonorphism $\operatorname{Aut}(A) / \operatorname{Im}(A) \mapsto S_{\Gamma}$. It is obvious that the above $\sigma$ is in $S_{\Gamma}$ and, in case of being well-defined, the map preserves multiplication. In order to prove that the assignment is a well-definef map, and hence establish our claim, we shall check that an element of $\operatorname{Aut}(A) / \operatorname{Inn}(A)$ is represented by a unique $\phi \in \operatorname{Aut}(A)$ that leaves invariant $\left\{e_{\nu} \mid \nu \in V(\Gamma)\right\}$. Indeed, if $\phi, \psi \in \operatorname{Aut}(A)$ leave invariant that basic set and $\phi \circ \psi^{-1}$ is inner, then an argument similar to the one followed in $c$ ) for $f$ shows that $\left(\phi \circ \psi^{-1}\right)\left(e_{\nu}\right)=e_{\nu}$, for every $\nu \in V(\Gamma)$. But then, by the proof of Thcorem $3, \phi \circ \psi^{-1}$ is incluced by a change of variables $f$ in $K[\Gamma]$ so that $\phi \circ \psi^{-1}=i_{\rho}(f)$. By part $\left.\mathrm{c}\right), f \equiv \mathrm{id}_{K(1)}$ and, consequently, $\phi \equiv \psi$ as desired.

Example 8. Assmme that the quiver of $A$ is

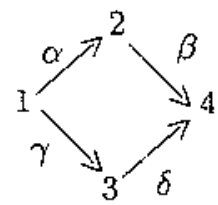

By the foregoing theorem, $V_{\Gamma} \cong K^{*} \times K^{*} \times K^{*} \times K^{*}$ and it is obvious that $S_{\Gamma}=\{1, \tau\} \cong \mathrm{C}_{2}$ (the multiplicative cyclic group with two elements), where $\tau$ is the transposition of vertices 2 and 3 . We shall determine $\operatorname{Aut}(A) / \operatorname{mn}(A)$ for some algebras having the above quiver.

a) $A=K[\Gamma]$. This case can be dealt with greater generality since, whatever the quiver, $V_{\Gamma}=V_{(r, p)}$ always and, when $\Gamma$ is also strongly acyclic, the group homomorphism $p: \operatorname{Aut}(A) / \operatorname{Im}(A) \multimap S_{\mathrm{I}}$ is surjective. In our particular situation it is casy to see that $p$ is also split, so that $\operatorname{Aut}(A) / \operatorname{Inn}(A) \cong\left(K^{*} \times K^{*} \times K^{*} \times K^{*}\right) \propto \mathrm{C}_{2}, \propto$ denoting a senidirect product. An element $\left[\left(c_{12}, c_{24}, c_{13}, c_{34}\right), T\right]$ of the second group represents the class modulo $\operatorname{Im}(A)$ of the composition $\left(c_{12}, c_{24}, c_{13}, c_{34}\right) \circ \tau$, where 
$\tau$ is the above mentioned transposition on the vertices and $\left(\begin{array}{c}\alpha \beta \gamma \delta \beta \\ \gamma \delta \alpha \beta\end{array}\right)$ on the arrows, while the quadruple $\left(c_{\nu \omega}\right)$ takes every arrow $\varepsilon: \nu \mapsto \omega$ to $c_{\nu \omega} \varepsilon$.

b) $A=K[\Gamma] /\langle\alpha \beta\rangle$. Here $V_{\Gamma}=V_{(\Gamma, \rho)}$ again and it is not hard to see that any $\phi \in \operatorname{Aut}(A)$ that leaves invariant the basic set of idempotents necessarily induces the identity on that set, thus proving that the image of $p: \operatorname{Aut}(A) / \operatorname{Inn}(A) \mapsto S_{\Gamma}$ is the trivial subgroup. Therefore $\operatorname{Aut}(A) / \operatorname{Inn}(A) \cong K^{*} \times K^{*} \times K^{*} \times K^{*}$.

c) $A=K[\Gamma] /\langle\alpha \beta, \gamma \delta\rangle . \quad V_{\Gamma}=V_{(\Gamma, \rho)}$ and $p$ is also split, so that the situation is exactly the same as in a).

d) $A=K[\Gamma] /\langle\alpha \beta-\gamma \delta\rangle$. In this case every change of variables (identified with the quadruple $\left.\left(c_{12}, c_{24}, c_{13}, c_{34}\right)\right)$ that leaves unaitered the adequate ideal $\langle\alpha \beta-\gamma \delta\rangle$ must necessarily satisfy $c_{12} c_{24}=c_{13} c_{34}$. Hence $V_{\langle\Gamma, \rho\rangle} \cong\left\{\left(c_{12}, c_{24}, c_{13}, c_{34}\right) \in K^{*} \times K^{*} \times K^{*} \times K^{*} / c_{12} c_{24}=c_{13} c_{34}\right\}$. On the other hand, $p$ is again a split epimorphism. Therefore Aut $/(A) \operatorname{Inn}(A)$ $\cong V_{(\Gamma, \rho)} \propto C_{2}$.

\section{References}

1. Anderson, F. W. And Fuller, K. R., "Rings and categories of modules," Springer-Verlag, 1974.

2. GABR1EL, P., "Auslander-Reiten sequences and representationfinite algebras. Representation Theory I," Springer, Lect. Not. Mat. 831, Proceedings, Carleton University 1979, 1980.

3. Pierce, R. S., "Associative algebras," Springer-Verlag, 1982.

Departamento de Matemáticas

Universidad de Murcia

30100 Espinardo

Murcia

SPAIN

Rebut el 7 de Gener de 1992 